\title{
OSCILLATION AND STABILITY IN A SIMPLE GENOTYPE SELECTION MODEL
}

BY

E. A. GROVE, V. LJ. KOCIĆ, G. LADAS (University of Rhode Island, Kingston, Rhode Island) AND

R. LEVINS (Harvard School of Public Health, Boston, Massachusetts)

Abstract. We study the oscillation, the stability, and the global attractivity of the simple genotype selection model

$$
y_{n+1}=\frac{y_{n} e^{\beta\left(1-2 y_{n-k}\right)}}{1-y_{n}+y_{n} e^{\beta\left(1-2 y_{n-k}\right)}}, \quad n=0,1, \ldots,
$$

where $\beta \in(0, \infty)$ and $k \in\{0,1,2, \ldots\}$.

1. Introduction. Our aim in this paper is to study the oscillation, the stability, and the global attractivity of the simple genotype selection model

$$
y_{n+1}=\frac{y_{n} e^{\beta\left(1-2 y_{n-k}\right)}}{1-y_{n}+y_{n} e^{\beta\left(1-2 y_{n-k}\right)}}, \quad n=0,1, \ldots,
$$

where

$$
\beta \in(0, \infty) \text { and } k \in\{0,1,2, \ldots\} .
$$

When $k=0$, Eq. (1.1) was introduced by May [9] as an example of a map generated by a simple model for frequency-dependent natural selection. Equation (1.1) gives the change in gene frequency between the $n$th generation and the next when the fitness function is $\exp (\beta(1-2 y))$.

When $k=0$, one possible derivation of May's model is as follows.

Consider the differential equation

$$
\dot{y}=y(1-y) \beta(1-2 y)
$$

for simple natural selection at a single locus with two allèles and without dominance.

Observe that Eq. (1.3) may be written in the form

$$
\frac{\dot{y}}{y(1-y)}=\beta(1-2 y)
$$

Received March 31, 1992.

1991 Mathematics Subject Classification. Primary 39A12.

Key words and phrases. Oscillation, stability, global attractivity, genotype selection model.

(C)1994 Brown University 
and that formal integration from $n$ to $n+1$ yields

$$
\left.\ln \frac{y(t)}{1-y(t)}\right|_{t=n} ^{t=n+1}=\int_{n}^{n+1} \beta(1-2 y(t)) d t .
$$

By setting $y(n)=y_{n}$ and by approximating the integral on the right by $\beta\left(1-2 y_{n}\right)$, Eq. (1.4) yields

$$
\frac{y_{n+1}}{1-y_{n+1}}=\frac{y_{n}}{1-y_{n}} e^{\beta\left(1-2 y_{n}\right)},
$$

from which Eq. (1.1) follows in the case $k=0$.

In Eq. (1.1), $y_{n}$ is the frequency of gene $A$ in a population, $1-y_{n}$ is the frequency of its allèle $a$, and $\beta\left(1-2 y_{n-k}\right)$ is the selection coefficient on $A$ that depends upon the gene frequency at some previous time. The use of a differential equation in population genetics is justified when there are overlapping generations and continuous reproduction. But when there are discrete generations, as in the case for species with one generation per year, then the use of difference equations is more appropriate.

The appearance of $y_{n-k}$ in the selection coefficient reflects the fact that the environment at the present time depends upon the activity of the population at some time in the past and that this in turn depends upon the gene frequency at that time. Since different genotypes act differently on the environment, the past genetic makeup can affect possible nesting sites, soil fertility, food supply, predators, and so on.

The symmetry of the model about $y=\frac{1}{2}$ and the absence of dominance are restrictive and were chosen to emphasize the role of the delay in determining the dynamics.

2. Preliminaries. In this section we gather some results which will be useful in the sequel. Let $\beta$ be a positive real number, let $k$ be a nonnegative integer, and consider the difference equation

$$
\left\{\begin{array}{l}
y_{n+1}=\frac{y_{n} e^{\beta\left(1-2 y_{n-k}\right)}}{1-y_{n}+y_{n} e^{\beta\left(1-2 y_{n-k}\right)}}, \quad n=0,1, \ldots ; \\
y_{-k}, y_{-k+1}, \ldots, y_{0} \in[0,1] .
\end{array}\right.
$$

Clearly $0, \frac{1}{2}$, and 1 are the only equilibrium solutions of Eq. (2.1). One can easily see by induction that $y_{n} \in[0,1]$ for all $n=0,1, \ldots$. Also if $y_{n}=0$ for some $N \in \mathbf{N}$, then $y_{n}=0$ for all $n \geq 0$; and if $y_{N}=1$ for some $N \in \mathbf{N}$, then $y_{n}=1$ for all $n \geq 0$. For this reason we often restrict our attention to the difference equation

$$
\left\{\begin{array}{l}
y_{n+1}=\frac{y_{n} e^{\beta\left(1-2 y_{n-k}\right)}}{1-y_{n}+y_{n} e^{\beta\left(1-2 y_{n-k}\right)}}, \quad n=0,1, \ldots ; \\
y_{-k}, y_{-k+1}, \ldots, y_{0} \in(0,1) .
\end{array}\right.
$$

Then clearly, $y_{n} \in(0,1)$ for all $n \geq-k$. By introducing the substitution

Eq. (2.2) becomes

$$
x=\frac{y}{1-y}=T(y) \text { for } 0<y<1,
$$

$$
\left\{\begin{array}{l}
x_{n+1}=x_{n} \exp \left(\beta \frac{1-x_{n-k}}{1+x_{n-k}}\right), \quad n=0,1, \ldots ; \\
x_{-k}, x_{-k+1}, \ldots, x_{0} \in(0, \infty) .
\end{array}\right.
$$


Note that $T(0)=0, T\left(\frac{1}{2}\right)=1, T(1-)=\infty$, and $T^{\prime}(y)=1 /(1-y)^{2}>0$, and so the dynamics of Eq. (2.2) are the same as those of Eq. (2.3) with $\frac{1}{2}$ replaced by 1 and 1 replaced by $\infty$. Clearly, $x_{n}>0$ for all $n \geq 0$. The unique positive equilibrium solution of Eq. (2.3) is $\bar{x}=1$, which corresponds to the equilibrium solution $\bar{y}=\frac{1}{2}$ of Eq. (2.2). After the substitution $x=e^{z}$, Eq. (2.3) becomes

$$
\left\{\begin{array}{l}
z_{n+1}-z_{n}+\beta \tanh \left(z_{n-k} / 2\right)=0, \quad n=0,1, \ldots ; \\
z_{-k}, z_{-k+1}, \ldots, z_{0} \in(-\infty, \infty)
\end{array}\right.
$$

Our substitution here is $z=\ln x=S(x)$ for $x \in(0, \infty)$. So as $S(0+)=-\infty$, $S(1)=0, S(\infty)=\infty$, and $S^{\prime}(x)=1 / x>0$, we see that the dynamics of Eq. (2.2) are also the same as those of Eq. (2.4) with 0 replaced by $-\infty, \frac{1}{2}$ replaced by 0 , and with 1 replaced by $\infty$.

For convenience we record some results from the literature which will be used in the sequel. The first result which is adapted from [6] gives sufficient conditions for the global asymptotic stability of a second-order difference equation. The second theorem, which is extracted from [7], gives sufficient conditions for the global attractivity of a nonlinear difference equation.

Theorem A [6]. Assume that $f \in \mathbf{C}[(0, \infty),(0, \infty)]$ is decreasing and that $u f(u)$ is increasing in $u$. Suppose that the difference equation

$$
x_{n+1}=x_{n} f\left(x_{n-1}\right), \quad n=0,1, \ldots,
$$

has a unique positive equilibrium $\bar{x}$. Then $\bar{x}$ is globally asymptotically stable.

THEOREM B [7]. Consider the difference equation

$$
x_{n+1}=x_{n} f\left(x_{n-k}\right), \quad n=0,1, \ldots,
$$

where $k$ is a positive integer and $f$ satisfies the following conditions:

(i) $f \in \mathbf{C}[[0, \infty),(0, \infty)], f(x)$ is nonincreasing in $x$;

(ii) Equation (2.6) has a unique positive equilibrium $\bar{x}$;

(iii) $(x f(x)-\bar{x})(x-\bar{x})>0$ for $x \neq \bar{x}$.

(iv) Set $F(x)=\bar{x}(f(x))^{k}$. Then $x=\bar{x}$ is the only solution of $F(F(x))=x$ in the interval $0 \leq x \leq F(0)$.

Then $\bar{x}$ is a global attractor of all positive solutions of Eq. (2.6).

The next theorem is adapted from Cull [1]. See also [2, 5, 10]. While it only treats the case $k=0$, it is very powerful in this case.

Theorem C [1]. Let $A \in(0, \infty]$, and let $g:[0, A) \rightarrow[0, A)$ be a continuous function. Suppose $g(0)=0, g(x)>0$ for $0<x<A$, and $g$ has a unique positive equilibrium $\bar{x}$. Suppose also that $g(x)>x$ for $0<x<\bar{x}$ and $g(x)<x$ for $\bar{x}<x<A$. Consider the difference equation

$$
\left\{\begin{array}{l}
x_{n+1}=g\left(x_{n}\right), \quad n=0,1, \ldots, \\
x_{0} \in(0, A) .
\end{array}\right.
$$

Then the following statements are true.

(i) If $g$ has no maximum in $(0, \bar{x})$, then $\bar{x}$ is a global attractor of all solutions of Eq. (2.7). Moreover, $\left\{x_{n}\right\}$ converges eventually monotonically to $\bar{x}$. 
(ii) Suppose $g$ has a maximum at a point $x_{m} \in(0, \bar{x})$ for which $g$ is strictly monotone decreasing for $x>x_{m}$. Then $\bar{x}$ is a global attractor of all solutions of Eq. (2.7) if and only if $x<g(g(x))$ for all $x \in\left[x_{m}, \bar{x}\right)$.

The next result is adapted from Fisher, Goh, and Vincent [3]. While it is also only valid for the case $k=0$, it is nevertheless a powerful tool in this case and, as we will see later, sometimes plays a role which is complementary to that of Theorem C. The function $V$ in Theorem D plays the role of a "discrete Lyapunov function".

Theorem D [3]. Let $A \in(0, \infty]$, and let $h \in \mathbf{C}[(0, A),(0, A)]$. Assume that $h$ has a unique fixed point $\bar{x} \in(0, A)$ and that there exists a function $V \in$ $\mathbf{C}[(0, A),[0, \infty)]$ such that the following conditions are satisfied:

(i) $V$ is nonincreasing in a neighborhood of 0 ;

(ii) $V$ is nondecreasing in a neighborhood of $A$;

(iii) $\Delta V(x) \equiv V(h(x))-h(x)<0$ for all $x \in(0, A)$ with $x \neq \bar{x}$.

Then $\bar{x}$ is a global attractor of all solutions of the equation

$$
\left\{\begin{array}{l}
x_{n+1}=h\left(x_{n}\right), \quad n=0,1, \ldots, \\
x_{0} \in(0, A)
\end{array}\right.
$$

The local stability analysis for biological models which are described by scalar nonlinear delay difference equations was presented by Levin and May [8].

Theorem E [8]. Assume that $q \in \mathbf{R}$ and $k \in\{0,1,2, \ldots\}$. Then the delay difference equation

$$
x_{n+1}-x_{n}+q x_{n-k}=0, \quad n=0,1, \ldots,
$$

is asymptotically stable if and only if $0<q<2 \cos \frac{k \pi}{2 k+1}$.

Theorems A-E have dealt with questions about global attractivity. The following theorems deal with questions about oscillation.

A sequence $\left\{x_{n}\right\}$ is said to oscillate if the terms $x_{n}$ are neither eventually all positive nor eventually all negative. Otherwise the sequence is called nonoscillatory. A sequence $\left\{x_{n}\right\}$ is said to oscillate about $\bar{x}$ if the sequence $\left\{x_{n}-\bar{x}\right\}$ oscillates.

The following basic linearized oscillation result is extracted from Györi and Ladas [4].

Theorem F [4]. Assume that $p \in(0, \infty)$ and $k \in\{0,1, \ldots\}$ with $p+k \neq 1$. Let $f \in C[\mathbf{R}, \mathbf{R}]$ be such that

$$
u f(u)>0 \text { for } u \neq 0, \quad \lim _{u \rightarrow 0} \frac{f(u)}{u}=1,
$$

and there exists a $\delta>0$ such that

$$
\text { either } f(u) \leq u \text { for } 0<u<\delta \text { or } f(u) \geq u \text { for }-\delta<u<0 .
$$

Then every solution of the nonlinear difference equation

$$
x_{n+1}-x_{n}+p f\left(x_{n-k}\right)=0, \quad n=0,1, \ldots,
$$

osciilates if and only if every solution of the linearized equation

$$
y_{n+1}-y_{n}+p y_{n-k}=0, \quad n=0,1, \ldots,
$$

oscillates. 
The following result is an immediate consequence of the fact that every solution of an autonomous, linear, homogeneous difference equation oscillates if and only if its characteristic equation has no positive roots.

Theorem $\mathrm{G}$ [4]. Assume that $p \in(0, \infty)$ and $k \in\{0,1,2, \ldots\}$. Then every solution of the delay difference equation

$$
y_{n+1}-y_{n}+p y_{n-k}=0, \quad n=0,1, \ldots,
$$

oscillates if and only if $p \geq 1$ if $k=0$ and $p>k^{k} /(k+1)^{k+1}$ if $k \geq 1$.

3. Oscillation. In this section we determine explicit necessary and sufficient conditions to ensure that every solution of the difference equation (2.2) oscillates about $\frac{1}{2}$.

TheOREM 3.1. Assume $\beta$ is a positive real number and $k$ is a nonnegative integer. Then the following statements are true:

(i) if $k=0$, then every solution of Eq. (2.2) oscillates about $\bar{y}=\frac{1}{2}$ if and only if $\beta>2$;

(ii) if $k \geq 1$, then every solution of Eq. (2.2) oscillates about $\bar{y}=\frac{1}{2}$ if and only if $\beta>2 k^{k} /(k+1)^{k+1}$.

Proof. In Sec. 2 we determined that the dynamics of Eq. (2.2) are equivalent to those of (2.4) with $\frac{1}{2}$ replaced by 0 . It is Eq. (2.4) that we shall utilize in the proof. First consider the case when $k+\beta / 2 \neq 1$. Since the function $f(u)=2 \tanh (u / 2)$ is continuous from $\mathbf{R}$ into $\mathbf{R}$ and such that

$$
u f(u)>0 \text { for } u \neq 0, \quad \lim _{u \rightarrow 0} \frac{f(u)}{u}=1,
$$

and

$$
f(u) \leq u \text { for } u \geq 0,
$$

by Theorem F, all solutions of Eq. (2.4) oscillate if and only if all solutions of the associated linearized equation (5.2) oscillate. By Theorem G, Eq. (5.2) oscillates if and only if

$$
\begin{aligned}
\text { either } & k=0 \text { and } \beta>2 \\
\text { or } & k \geq 1 \text { and } \beta>2 \frac{k^{k}}{(k+1)^{k+1}} .
\end{aligned}
$$

Finally when $k+\beta / 2=1$, which is equivalent to $k=0$ and $\beta=2$, the result follows from Theorem $\mathrm{C}(\mathrm{i})$.

4. Stability when $k=0$. In this section we study the stability behavior of the difference equation (2.1) when $k=0$. For $0<x<1$, set

$$
g(x)=\frac{x e^{\beta(1-2 x)}}{1-x+x e^{\beta(1-2 x)}} .
$$

Since $g^{\prime}(0)=g^{\prime}(1)=e^{\beta}>1$, it follows by linearized stability analysis that 0 and 1 are unstable equilibrium solutions of Eq. (2.1) with $k=0$. 
THEOREM 4.1. Let $\beta>0$ and consider the difference equation

$$
\left\{\begin{array}{l}
y_{n+1}=\frac{y_{n} e^{\beta\left(1-2 y_{n}\right)}}{1-y_{n}+y_{n} e^{\beta\left(1-2 y_{n}\right)}}, \quad n=0,1, \ldots, \\
y_{0} \in(0,1) .
\end{array}\right.
$$

Then the following statements are true:

(i) if $0<\beta \leq 4$, then the equilibrium solution $y_{n}=\frac{1}{2}$ is globally asymptotically stable;

(ii) if $\beta>4$, then the equilibrium solution $y_{n}=\frac{1}{2}$ is unstable.

Proof. The proof will be established in a series of three lemmas. Lemma 4.1 was given in [9].

LEMMA 4.1. If $0<\beta<4$, then $y_{n}=\frac{1}{2}$ is an asymptotically stable solution of Eq. (4.2); while if $\beta>4$, then $y_{n}=\frac{1}{2}$ is an unstable solution of Eq. (4.2).

Proof. $g^{\prime}\left(\frac{1}{2}\right)=1-\beta / 2$ and so the result follows by linearized stability.

Lemma 4.2. Let $0<\beta \leq 2$. Then $y_{n}=\frac{1}{2}$ is a global attractor of Eq. (4.2).

Proof. We wish to apply Theorem C. Consider $g:[0,1) \rightarrow[0,1)$ where $g$ is defined by (4.1). Then $g$ is continuous, $g(0)=0, g(x)>0$ if $0<x<1$, and $g$ has exactly one positive fixed point $\bar{x}=\frac{1}{2}$. Note that $g\left(\frac{1}{4}\right)>\frac{1}{4}$ and so $g(x)>x$ if $0<x<\frac{1}{2}$. Similarly, $g\left(\frac{3}{4}\right)<\frac{3}{4}$ from which it follows that $g(x)<x$ if $\frac{1}{2}<x<1$. Finally, $g^{\prime}\left(x_{m}\right)=0$ if and only if $x_{m}=\frac{1}{2}\left(1 \pm\left(1-\frac{2}{\beta}\right)^{1 / 2}\right)$. But $1-\frac{2}{\beta}>0$ is equivalent to $\beta>2$ and we have assumed that $0<\beta \leq 2$. The result follows by Theorem $\mathrm{C}(\mathrm{i})$.

LEMMA 4.3. Let $2<\beta \leq 4$. Then $y_{n}=\frac{1}{2}$ is a globally asymptotically stable solution of Eq. (4.2).

Proof. We shall use Theorem D. Consider $g:(0,1) \rightarrow(0,1)$ where $g$ is defined by Eq. $(4.1)$, and let $V:(0,1) \rightarrow[0, \infty)$ be given by $V(x)=\left(x-\frac{1}{2}\right)^{2}$. Clearly $V$ is continuous, decreasing on $\left(0, \frac{1}{2}\right)$ and increasing on $\left(\frac{1}{2}, 1\right)$. If $0<x<1$,

$$
\begin{aligned}
\Delta V(x) & =V(g(x))-V(x)=\left(\frac{x e^{\beta(1-2 x)}}{1-x+x e^{\beta(1-2 x)}}-\frac{1}{2}\right)^{2}-\left(x-\frac{1}{2}\right)^{2} \\
& =-x e^{\beta(1-2 x)}\left[\frac{e^{\beta(1-2 x)}}{1-x+x e^{\beta(1-2 x)}}-1\right]\left[\frac{-x}{1-x+x e^{\beta(1-2 x)}}+\frac{(1-x)}{e^{\beta(1-2 x)}}\right] .
\end{aligned}
$$

Observe that

$$
\frac{e^{\beta(1-2 x)}}{1-x+x e^{\beta(1-2 x)}}-1=\frac{(1-x)\left(e^{\beta(1-2 x)}-1\right)}{1-x+x e^{\beta(1-2 x)}} \begin{cases}>0 & \text { if } 0<x<\frac{1}{2} \\ =0 & \text { if } x=\frac{1}{2} \\ <0 & \text { if } \frac{1}{2}<x<1\end{cases}
$$

Also

$$
\frac{-x}{1-x+x e^{\beta(1-2 x)}}+\frac{(1-x)}{e^{\beta(1-2 x)}}=\frac{x(1-x)\left(1+\frac{x}{1-x} e^{\beta(1 / 2-x)}\right)\left(\frac{1-x}{x}-e^{\beta(1 / 2-x)}\right)}{\left(1-x+x e^{\beta(1-2 x)}\right) e^{\beta(1-2 x)}} .
$$


Since $0<x<1$, we need only to determine the sign of $G(x)=\frac{1-x}{x}-e^{\beta(1 / 2-x)}$.

We shall first show that $G(x)>0$ if $0<x<\frac{1}{2}$. Note that $G\left(\frac{1}{2}\right)=0$, and so it suffices to show that $G^{\prime}(x)<0$ for $0<x<\frac{1}{2}$. Now

$$
G^{\prime}(x)=-\frac{1}{x^{2}} e^{-\beta x}\left(e^{\beta x}-x^{2} \beta e^{-\beta / 2}\right) .
$$

Set $H(x)=e^{\beta x}-x^{2} \beta e^{\beta / 2}$. Then $H(0)=1$ and $H\left(\frac{1}{2}\right)=e^{\beta / 2}\left(1-\frac{\beta}{4}\right) \geq 0$. It suffices to show that if $0<x<\frac{1}{2}$ and $H^{\prime}(x)=0$, then $H(x)>0$. Note that $H^{\prime}(x)=\beta e^{\beta x}-2 x \beta e^{\beta / 2}$. Suppose $H^{\prime}(x)=0$, where $0<x<\frac{1}{2}$. Then

$$
0=H^{\prime}(x)=\beta e^{\beta x}-2 x \beta e^{\beta / 2}=\beta\left(e^{\beta x}-2 x e^{\beta / 2}\right)
$$

and so $e^{\beta x}=2 x e^{\beta / 2}$. Hence $H(x)=x e^{\beta / 2}(2-x \beta)>0$ since $0<x<\frac{1}{2}$ and $\beta \leq 4$.

We shall next show that $G(x)<0$ if $\frac{1}{2}<x<1$. Because $G\left(\frac{1}{2}\right)=0$, it suffices to show that $G^{\prime}(x)<0$ for $\frac{1}{2}<x<1$. But $G^{\prime}(x)=-H(x) / x^{2}$, and so we need only show that $H(x)>0$, for $\frac{1}{2}<x<1$. Now $H\left(\frac{1}{2}\right)=e^{\beta / 2}\left(1-\frac{\beta}{4}\right) \geq 0$, and so it suffices to show $H^{\prime}(x)>0$ for $\frac{1}{2}<x<1$. But $H^{\prime}(x)=\beta e^{\beta x}-2 x \beta e^{\beta / 2}$ and in particular, $H^{\prime}\left(\frac{1}{2}\right)=0$. This means that the claim will be established if we can show that

$$
H^{\prime \prime}(x)>0 \text { for } \frac{1}{2}<x<1 .
$$

Note that since $\beta>2, H^{\prime \prime}(x)=\beta^{2} e^{\beta x}-2 \beta e^{\beta / 2}>0$, and so $G(x)$ is negative for $\frac{1}{2}<x<1$.

Hence $\Delta V(x)<0$ for all $x \in(0,1)$ with $x \neq \frac{1}{2}$, and so we see by Theorem $D$ that $y_{n}=\frac{1}{2}$ is a global attractor of Eq. (4.2). Thus by Lemma 4.1 we see that if $0<\beta<4$, then $y_{n}=\frac{1}{2}$ is a globally asymptotically stable equilibrium solution of Eq. (4.2). Finally if $\beta=4$ and $x_{0} \in(0,1)$ with $x_{0} \neq \frac{1}{2}$, then the fact that $\Delta V(x)<0$ for $x \in(0,1)$ with $x \neq \frac{1}{2}$ implies

$$
\left|x_{0}-\frac{1}{2}\right|>\left|x_{1}-\frac{1}{2}\right|>\cdots
$$

and so $y_{n}=\frac{1}{2}$ is a globally asymptotically stable equilibrium solution in the case $\beta=4$ also.

REMARK 4.1. It is interesting to note that Theorem $C$ applies only in the case $0<\beta \leq 2$ while Theorem $\mathrm{D}$ applies only in the case $2<\beta \leq 4$.

5. Stability when $k \geq 1$. Consider the delay difference equation (2.1) where $\beta$ is a positive real number and $k$ is a positive integer. We shall first consider the local stability of Eq. (2.1). There are three linearized equations associated with Eq. (2.1); these equations deal with the equilibrium solutions $0, \frac{1}{2}$, and 1 . The linearized equation associated with the equilibrium solution 0 is

$$
w_{n+1}-e^{\beta} w_{n}=0, \quad n=0,1, \ldots
$$

The linearized equation associated with the equilibrium solution $\frac{1}{2}$ is

$$
w_{n+1}-w_{n}+\frac{\beta}{2} w_{n-k}=0, \quad n=0,1, \ldots
$$

The linearized equation associated with the equilibrium solution 1 is again Eq. (5.1). 
LEMMA 5.1.

(i) The equilibrium solution $\bar{y}=0$ of Eq. (2.1) is unstable.

(ii) The equilibrium solution $\bar{y}=\frac{1}{2}$ of Eq. (2.1) is locally asymptotically stable if $0<\beta<4 \cos \frac{k \pi}{2 k+1}$ and is unstable if $\beta>4 \cos \frac{k \pi}{2 k+1}$.

(iii) The equilibrium solution $\bar{y}=1$ of Eq. (2.1) is unstable.

Proof. Statements (i) and (iii) follow immediately from linearized stability while statement (ii) follows from linearized stability and Theorem E.

Theorem 5.1. Consider the delay difference equation (2.2) where $\beta$ is a positive real number and $k$ is a positive integer. Then the following statements are true.

(i) Suppose $k=1$. Then the equilibrium solution $\bar{y}=\frac{1}{2}$ is globally asymptotically stable if $0<\beta \leq 2$ and is unstable if $\beta>2$.

(ii) Suppose $k \geq 2$. Then the equilibrium solution $\bar{y}=\frac{1}{2}$ is globally asymptotically stable if $0<\beta \leq \frac{2}{k}$.

Proof. We wish to use Theorems A and B. It is more convenient to study the stability nature of the positive equilibrium solution $\bar{x}=1$ of the equivalent difference equation (2.3). For $x \geq 0$, define $f(x)$ by the formula

$$
f(x)=\exp \left(\beta \frac{1-x}{1+x}\right) .
$$

Clearly, when $k=1$ and $0<\beta \leq 2, f$ satisfies all the hypotheses of Theorem A and so $\bar{x}=1$ is a globally asymptotically stable equilibrium of Eq. (2.3). Also linearized stability shows that when $k=1$ and $\beta>2, \bar{x}=1$ is an unstable equilibrium of Eq. (2.3). The proof of (i) is complete.

Now suppose $k \geq 2$. The hypotheses (i), (ii), and (iii) of Theorem B are clearly satisfied. Set

$$
F(x)=(f(x))^{k}=\exp \left(k \beta \frac{1-x}{1+x}\right) .
$$

It suffices to verify that the equation $F(F(x))=x$ has the unique nonnegative solution $\bar{x}=1$. To this end, consider the difference equation

$$
\left\{\begin{array}{l}
v_{n+1}=F\left(v_{n}\right), \quad n=0,1, \ldots, \\
v_{0} \in[0, \infty) .
\end{array}\right.
$$

We shall show that if $0<\beta \leq \frac{2}{k}$, then 1 is a global attractor of Eq. (5.3) and so the proof that $\bar{y}=\frac{1}{2}$ is a global attractor will be complete. So assume $0<\beta \leq \frac{2}{k}$. Since $F(x)$ is a decreasing function on $[0, \infty)$, it follows that $\bar{v}=1$ is the unique equilibrium solution of Eq. (5.3). Clearly, any solution of Eq. (5.3) has the property that $v_{n}>0$ for $n \geq 1$. After the substitution $v_{n}=e^{u_{n}}$, Eq. (5.3) is transformed into the equivalent equation

$$
\left\{\begin{array}{l}
u_{n+1}=k \beta \frac{1-e^{u_{n}}}{1+e^{u_{n}}}, \quad n=0,1, \ldots, \\
u_{0} \in \mathbf{R} .
\end{array}\right.
$$


What we shall actually prove is that the equilibrium solution $\bar{u}=0$ is a global attractor of Eq. (5.4). Consider the function

$$
G(u)=k \beta \frac{1-e^{u}}{1+e^{u}}=-k \beta \tanh \frac{u}{2} .
$$

Observe that $G(0)=0$,

$$
|G(u)|=|G(|u|)|,
$$

and that

$$
|G(u)|<\frac{k \beta}{2}|u| \leq|u| \text { for } u \neq 0
$$

Hence from (5.4)

$$
\left|u_{n+1}\right|=\left|G\left(u_{n}\right)\right|<\left|u_{n}\right|, \quad n=0,1, \ldots,
$$

from which the result follows.

Finally, in order to apply Lemma 5.1 to complete the proof of statement (ii), we must show

$$
\frac{2}{k}<4 \cos \frac{k \pi}{2 k+1}
$$

for every integer $k \geq 2$. With this in mind, consider the inequality

$$
\frac{2}{x}<4 \cos \frac{x \pi}{2 x+1} \text { for } x>1 \text {. }
$$

The change of variables $t=\pi /(2 x+1)$ transforms Eq. (5.5) into the equivalent inequality

$$
\frac{2 t}{\pi-2 t}<\sin t, \quad 0<t<\pi / 6 .
$$

Now $2 t /(\pi-2 t)$ is a strictly concave-up function on $(0, \pi / 6)$ and $\sin t$ is a strictly concave-down function on $(0, \pi / 6)$. The proof of inequality (5.6) (and hence of Theorem 5.1) follows from the observation that

$$
\frac{2 t}{\pi-2 t}=\sin t \text { for } t=\pi / 6
$$

ReMARK 5.1. In view of Theorem 5.1, the condition $0<\beta \leq 2$ for global asymptotic stability when $k=1$ is "sharp". Our condition for global asymptotic stability (or even global attractivity) is probably far from sharp when $k \geq 2$. In this case we conjecture the condition should be

$$
0<\beta \leq 4 \cos \frac{k \pi}{2 k+1} \text {. }
$$

REMARK 5.2. According to May $[9$, p. 540] "for frequency-dependent selective forces so strong that $\beta>4$, the equilibrium point $\bar{y}=\frac{1}{2}$ of Eq. (1.3) when $k=0$ gives way to a stable 2-point cycle, ... and the 2-cycle never becomes unstable".

The analytical justification of the above remark is the starting point of a forthcoming paper by the authors. 


\section{REFERENCES}

[1] P. Cull, Global stability of population models, Bull. Math. Biol. 43, 47-58 (1981)

[2] P. Cull, Stability of discrete one-dimensional population models, Bull. Math. Biol. 50, 67-75 (1988)

[3] M. E. Fisher, B. S. Goh, and T. L. Vincent, Some stability conditions for discrete-time single species models, Bull. Math. Biol. 41, 861-875 (1979)

[4] I. Györi and G. Ladas, Oscillation Theory of Delay Differenial Equations with Applications, Clarendon Press, Oxford, 1991

[5] Y. Huang, A note on stability of discrete population models, Math. Biosci. 95, 189-198 (1989)

[6] J. H. Jaroma, V. Lj. Kocić, and G. Ladas, Global asymptotic stability of a second-order difference equation, Partial Differential Equations (J. Wiener and J. K. Hale, eds.), Pitman Research Notes in Mathematics Series, no. 273, Longman Scientific and Technical, 1992, pp. 80-84

[7] V. Lj. Kocić and G. Ladas, Global attractivity in nonlinear delay difference equations, Proc. Amer. Math. Soc. 115, 1083-1088 (1992)

[8] S. Levin and R. May, A note on difference-delay equations, Theoret. Population Biol. 9, 178-187 (1976)

[9] R. M. May, Nonlinear problems in ecology and resource management, Course 8 in Chaotic Behaviour of Deterministic Systems (G. Iooss, R. H. G. Helleman, and R. Stora, eds.), North-Holland, Amsterdam, 1983

[10] G. Rosenkranz, On global stability of discrete population models, Math. Biosci. 64, 227-231 (1983) 